\title{
Near Centimeter-scale FT-MIR Spectrometer based on NZIM Perfect Absorption using Inverted TanCirc Conformal Mapping Geometry
}

Dwight W. Swett ( $\sim$ dwight.swett@aramcoamericas.com )

Aramco Services Company, Aramco Research Center

\section{Research Article}

Keywords: Centimeter-scale FT-MIR Spectrometer, NZIM Perfect Absorption, Inverted TanCirc Conformal Mapping Geometry

Posted Date: November 16th, 2021

DOI: https://doi.org/10.21203/rs.3.rs-1047302/v1

License: (c) (i) This work is licensed under a Creative Commons Attribution 4.0 International License.

Read Full License 


\title{
Near Centimeter-scale FT-MIR Spectrometer based on NZIM Perfect Absorption using Inverted TanCirc Conformal Mapping Geometry
}

\author{
Dwight W. Swett ${ }^{1, \mathrm{a}}$ \\ ${ }^{1}$ Aramco Services Company, Aramco Research Center-Houston, TX 77084, USA \\ adwight.swett@aramcoamericas.com
}

\begin{abstract}
A long sought objective of MEMS research within the oil \& gas industry has been the realization of "FT-IR on a chip," which could hold the potential to migrate laboratory grade chemical spectroscopy into downhole sensor suites. A fundamental obstacle to this research has been the cooling demands of conventional technologies which conflict with the miniaturized sensor volumes required in downhole logging systems and environmental conditions that routinely exceed $125{ }^{\circ} \mathrm{C}$. Near centimeter scale spectroscopic devices are required by a majority of downhole sensor suites, which stands in stark contrast to multiple-decimeter size conventional devices. Here we report a near-centimeter scale FT-MIR ATR spectrometer compatible with downhole volumetric and temperature constraints. The spectrometer is based upon a high-temperature broadband mid-infrared metasurface detector/source combination derived from a geometric inversion of a set of conformal mapping contours. The metasurface elemental structure is derived from a geometric inversion of the canonical TanCirc conformal mapping contours and was found to exhibit a near zero index metamaterial (NZIM) behavior over a spectral range of interest for downhole chemical spectroscopy. The NZIM properties of the metasurface lead to an absorption phenomenon characterized by surface plasmon resonances which confine the absorption mechanism within the ultrathin $(\lambda / 300)$ metasurface plane and make the absorption properties of the microbolometer design relatively insensitive to the material properties of the remaining laminae. This unusual feature allows the metasurface to be integrated on a single $\mathrm{VO}_{2}$ material thermometric layer and operated at elevated downhole temperatures despite corresponding to the VO2 metal-insulator-transition (MIT) region. Within this transition region however the $\mathrm{VO} 2$ layer exhibits a substantially beneficial property in that the $\mathrm{VO}_{2}$ layer exhibits more than an order of magnitude enhancement in its ambient thermometric properties, leading to an uncooled microbolometer design with predicted maximum detectivity $\mathrm{D}^{*}=1.5 \times 10^{10} \mathrm{~cm} \sqrt{\mathrm{Hz}} / \mathrm{W}$ and noise equivalent difference temperature NEDT of $1 \mathrm{mK}$ at a modulation frequency of $500 \mathrm{~Hz}$. A sub-millimeter scale thermal infrared source with intrinsic mid-infrared band-limited emission is formed from the same cellular geometric building block, enabling the spectrometer miniaturization. These performance parameters compare well with lower tier laboratory grade FT-MIR spectroscopic instruments and could represent a significant step in the effort towards deploying miniaturized high-temperature mid-infrared spectroscopy into oilfield downhole logging applications.
\end{abstract}

\section{Introduction}

In the oil \& gas industry, the potential to migrate existing FT-IR technology into downhole logging application holds important benefits in the identification and analysis of in situ hydrocarbons particularly with respect to the mid-infrared regime which has been used for chemical analysis and quantification of saturate, aromatic, resin, and asphaltenic (SARA) components. However, the size and thermal control constraints of downhole sensor suites have to date confronted efforts to develop this technology for downhole integration. For instance, in downhole logging while drilling (LWD) operations temperatures can exceed 175 ${ }^{\circ} \mathrm{C}$ with $200 \mathrm{MPa}$ wellbore pressure, and sensor packages less than a 4-5 centimeters in diameter are typical. Production logging conditions can be generally more benign, at less than $125^{\circ} \mathrm{C}$ and $100 \mathrm{MPa}$, but packaging constraints are even more stringent with sensors less than a couple of centimeters in diameter needed. Downhole applications are effectively incompatible with integration of cryogenic cooling systems characteristic of laboratory grade detectors, as well as the thermal dissipation loads created by conventional Blackbody infrared sources. Thus, realization of the concept of "FT-IR on a chip" for downhole application will invariably require the development of unconventional technologies.

Many of the on-chip spectrometers proposed to date have been dispersive designs which use gratings or microresonators to separate certain wavelengths of broadband light in a spectral range and direct each wavelength individually to a detector. These designs suffer from inherent signal-to-noise ratio (SNR) penalties due to spreading the input light beam over many spectral channels making these unsuitable for downhole chemical analysis applications. Non-dispersive instruments, such as FT-IR spectrometers, do not separate out the individual wavelengths of the broadband light source and as a result retain 
what is called the multiplex advantage with a significantly higher SNR compared to dispersive designs. Lab benchtop FT-IR spectrometers utilize this nondispersive approach in an optical design incorporating moveable mirrors to generate an optical path length (OPL) difference leading to an interferogram that can be processed by Fourier transform (FT) techniques into a wavelength spectrum. Proposed on-chip FT-IR spectrometer designs have been unable to translate the high-resolution laboratory optical design into a miniaturized package and have focused principally on the near infrared regime using off-chip infrared sources, as described by Sabry et. $\mathrm{al}^{1}$. Other on-chip FT-IR interferometers have been proposed that rely on thermo-optic or electro-optic modulation to change the OPL in a waveguide, such as described by $\mathrm{Zheng}^{2}$ using a tunable micro-ring resonator filter, or Souza ${ }^{3}$ using a thermo-optic nonlinearity and dispersion correction. The very small refractive index modifications produced by these modulation effects, however, result in large device footprints and constrain the practically attainable spectral resolution to tens of $\mathrm{cm}^{-1}$ in wave number, far inferior to lab benchtop devices and insufficient for downhole chemical spectroscopy applications which need on the order of $10 \mathrm{~cm}^{-1}$ resolution for discrimination of the various elements and compounds of interest. Kita et. $\mathrm{al}^{4}$ in a group from MIT recently proposed an on-chip concept that utilizes a so-called digital Fourier transform (dFT) interferometer that acquires high-resolution spectra via time-domain modulation of a reconfigurable Mach-Zehnder interferometer and claims the multiplex advantage. Noise suppression is accomplished through the adaptation of machine learning regularization techniques for spectrum reconstruction. Their design for a 64-channel dFT interferometer has approximately $17 \times 20 \mathrm{~mm}^{2}$ in footprint, and the machine learning techniques provided on the order of $1 \mathrm{~cm}^{-1}$ resolution in a narrow short wavelength bandwidth between $1.55-1.57 \mu \mathrm{m}$. The $\mathrm{dFT}$ spectrometer volume lacks accommodation for light source, measurement sample interface, and the beam handling optics.

Conventional downhole reservoir fluid analysis is conducted either by formation sampling \& testing during the open-hole stage prior to casing (during drilling or immediately after the well is drilled), or by wellbore production logging (after the well is cased and perforated). A variety of sensors may be utilized in these operations, with generally a wider selection of technologies being available for formation sampling \& testing than is utilized in the production logging phase largely due to size constraints imposed by casing and production equipment. As a result production logs lack many types of data important to zonal fluid composition analysis once the reservoir enters production. The more typical information retrieved from a production logging sensor suite is related to estimating water, oil, and gas volume fractions and relies on a priori knowledge or assumption of much of these constituent properties. Direct measurement types of technologies such as visco-acoustic (measurement of fluid density, sound speed, and viscosity) and chemical spectroscopy (elemental properties characterization) would enhance the data available from production logs and allow other analyses such as SARA quantification through measurement of the wellbore fluid composition. The preferred chemical spectroscopy method for downhole application would be Fourier transform mid-infrared (FT-MIR) due to the high modulation frequencies that can be achieved, which would support real-time fluid properties measurement. However, the challenge confronting realization of this concept for in situ downhole application remains in the limitations of the state of the art in infrared detector technologies and blackbody radiation sources. Fundamentally these limitations derive from poor detectivities at the modulation frequencies and elevated temperatures associated with downhole logging environments, and the thermal management implications of integrating a full spectrum blackbody radiation source into a sensor suite at downhole temperatures. Conventional FT-MIR systems that offer the needed detectivity at sample rates rapid enough to be of interest for real-time analysis are based upon superconductivity and/or actively cooled detector technologies that typically operate over a narrow bandwidth of the IR spectrum, and in conjunction rely upon full spectrum blackbody radiation sources that would create problematic downhole thermal management issues. To enable an FT-MIR concept for in situ downhole logging, a completely different type of detector/source technology is needed that (i) requires no active cooling in order to achieve the detectivity which would support real time analysis sample rates, (ii) operates over the full MIR bandwidth of interest for discrimination of reservoir chemicals, and (iii) develops a minimal thermal footprint compatible with the constraints of downhole logging operations. This type of technology would fall well outside the capabilities of the current state of the art for uncooled detector technologies and infrared sources, but could well be adaptable to novel approaches such as electromagnetic metamaterials. In the following sections we describe a miniaturized FT-MIR spectrometer which leverages the unusual perfect absorbtion properties of a near-zero index metasurface to achieve significant progress towards this goal for downhole application. 

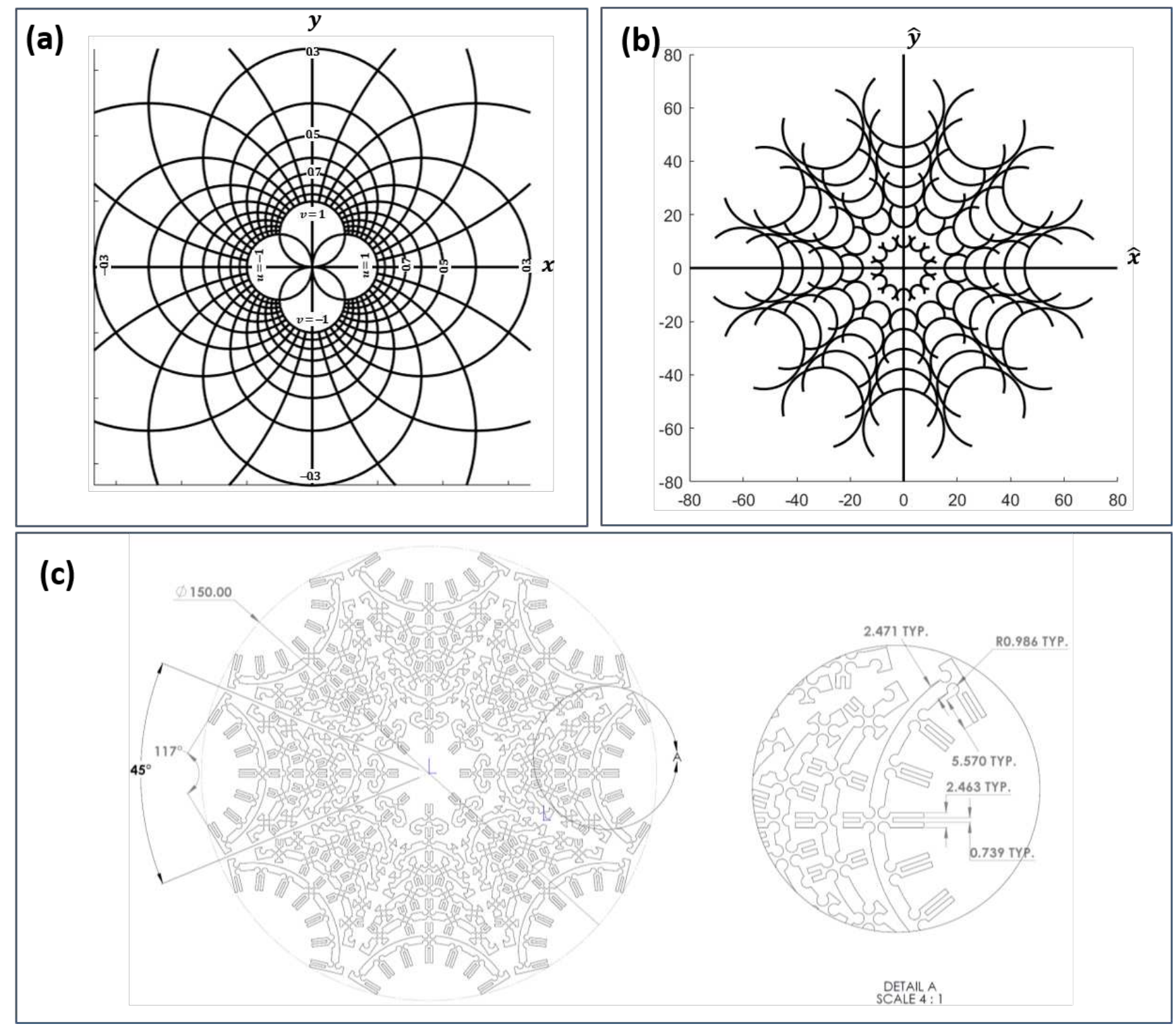

Figure 1. Development of the metasurface geometry, (a) base TanCirc conformal mapping contours ${ }^{5}$, (b) geometric inversion of base conformal contours, (c) final metasurface geometry formed along inverted contours (dimensions in microns).

\section{Inverted TanCirc Metasurface}

Fundamentally, the miniaturized downhole spectrometer is built upon a perfect metasurface absorber for the uncooled microbolometer detector and thermal infrared source. In a previous work Swett ${ }^{6}$ described how a broadband electromagnetic absorption could be achieved from a metamaterial built upon complex sub-cellular geometries derived from geometric inversion of a Rhodonea conformal mapping set of contours. Here we use a similar approach and report an electromagnetic metamaterial derived from a geometric inversion of the canonical TanCirc conformal mapping contours leading to a metamaterial also with near zero index metamaterial (NZIM) behavior and broad perfect absorption bandwidth. The absorption mechanism is confined to the ultrathin metasurface and makes the absorption properties of the detector relatively independent of the material properties of the remaining materials that may comprise the microbolometer. This unusual feature is significant for integration into downhole systems. It now allows integration of the perfect metasurface absorber with a common thermometric material layer, undoped $\mathrm{VO}_{2}$, that exhibits a metal-insulator-transition (MIT) region, that is to say, in the region where the thermometric material is transitioning from an insulator to an electrically conductive metal. For a thermometric material such as undoped $\mathrm{VO}_{2}$, in this metal-insulator-transition state the thermometric properties improve significantly from room temperature. Our approach is based on integration of the perfect metasurface absorber in a microbolometer construction with a single $\mathrm{VO}_{2}$ 
material thermometric layer temperature controlled to $60^{\circ} \mathrm{C}$ resulting in about 50X enhancement in the thermometric properties compared to room temperature. With elevated temperature control detectivity performances are expected that conventionally require decreasing the cavity temperature to cryogenic conditions $\left(<-200^{\circ} \mathrm{C}\right)$.

The metasurface geometry is based upon geometric inversion of the canonical TanCirc conformal mapping contours defined by the relations which transform Cartesian coordinate space to a new virtual domain described by (Field Theory handbook ${ }^{5}$ ):

$$
\begin{aligned}
& x=\frac{u}{u^{2}+v^{2}} \\
& y=\frac{v}{u^{2}+v^{2}}
\end{aligned}
$$

The geometric inversion of these conformal contours is developed using:

$$
\begin{gathered}
\hat{x}=\frac{u}{u^{2}+v^{2}}-\frac{2}{u} \\
\hat{y}=\frac{v}{u^{2}+v^{2}}
\end{gathered}
$$

which gives a new set of non-conformal contours that in effect 'invert' the original TanCirc geometry from inside-out. A graphical illustration of the geometric inversion process from the original TanCirc conformal contours transformed to the inverted contours and eventually to a metasurface geometry is shown in Figure 1. The details of the subcell of the metasurface geometry is depicted in the lower righthand of Figure 1.
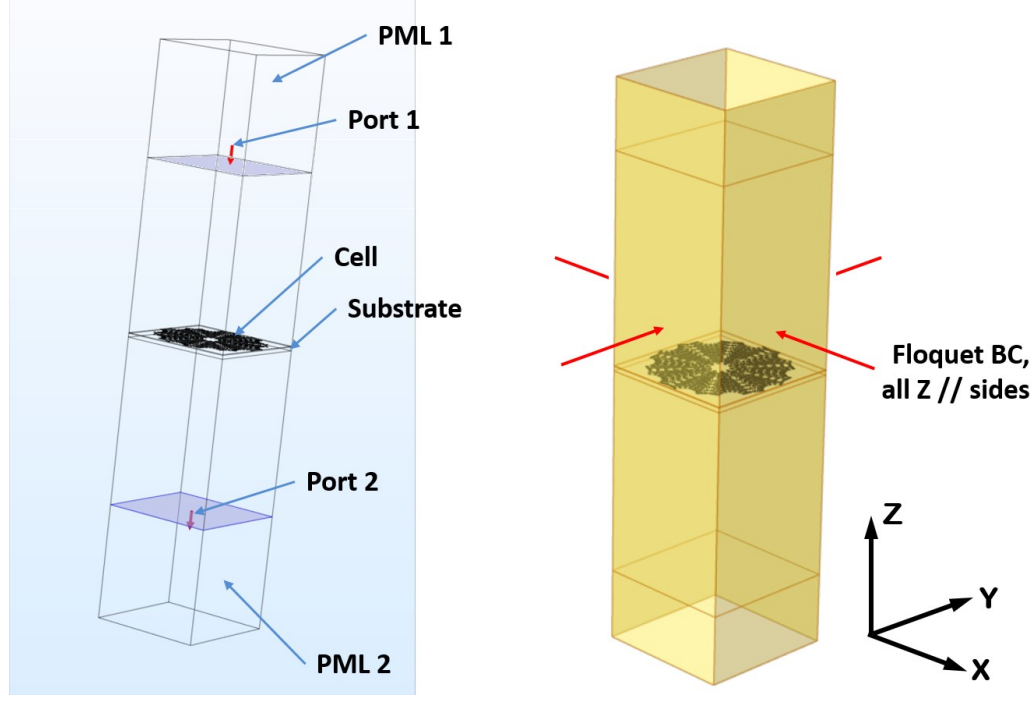

Figure 2. Scattering parameters retrieval finite element model.

The electromagnetic effective material properties were calculated using conventional scattering parameters (S-parameters) retrieval techniques in a 3D finite element analysis of the inverted TanCirc elemental cell with the commercially available Comsol ${ }^{\circledR}$ MultiPhysics 5.4 finite element analysis software package. The simulation model consisted of a 3D periodic waveguide geometry integrating the metasurface pattern centrally located between input and output ports as illustrated in Figure 2. The metasurface is modeled as a 2D Gold material pattern of $27 \mathrm{~nm}$ thickness using the surface transition boundary condition feature of the Comsol ${ }^{\circledR}$ MultiPhysics software package. The metasurface was modeled with the geometry as described in Figure $1 \mathrm{c}$ with a $150 \mu \mathrm{m}$ circumscribed diameter. The metasurface material is modeled as Gold with an electrical permittivity that is frequency dependent following a Drude $\operatorname{model}^{7}$ of the form:

$$
\varepsilon_{\text {gold }}(\omega)=\left[1-\frac{\omega_{p}^{2}}{\omega^{2}+\gamma^{2}}\right]+j\left[\frac{\omega_{p}^{2} \gamma}{\omega^{3}+\omega \gamma^{2}}\right]
$$

in which $\omega_{p}=2.15 \times 10^{15} \mathrm{~Hz}$ is the plasma frequency and $\gamma=17.14 \times 10^{12} \mathrm{~Hz}$ is the electromagnetic damping frequency. For the S-parameters retrieval, the metasurface pattern is modeled as free suspended in the vacuum waveguide. For the microbolometer infrared absorption simulations the $27 \mathrm{~nm}$ Gold metasurface pattern is modeled on the surface of a 3D thermometric layer of $135 \mathrm{~nm}$ thick $\mathrm{VO}_{2}$ material with a $500 \mathrm{~nm}$ spaced PEC boundary condition. The $\mathrm{VO}_{2}$ material was 
modeled with constant properties of relative permittivity $\varepsilon_{r}=10$, relative permeability $\mu_{r}=1$, and electrical conductivity $\sigma=2881 \mathrm{~S} / \mathrm{m}$ corresponding to material properties within the metal-insulator-transition region at $60^{\circ} \mathrm{C}$. The cross-section of the waveguide coincides with the thermometric layer dimensions. The remainder of the waveguide has the properties of free space (vacuum). The waveguide is excited with an incident transverse magnetic (TM) wave from Port 1 according to:

$$
\begin{array}{r}
\mathbf{H}=H_{0}\left[\begin{array}{lll}
0 & H_{y} & 0
\end{array}\right] \\
H_{y}=e^{j k x} e^{j k y}
\end{array}
$$

in which $k=\omega / c$ is the wavenumber in free space with $\omega$ the electromagnetic frequency and $\mathrm{c}$ the speed of light in vacuum. A second Port 2 is located at the opposite end of the waveguide placed symmetrically about the metasurface plane from Port 1 . To account for plane wave interaction with the periodic structure a set of Floquet periodic boundary conditions are imposed on the waveguide boundaries perpendicular to the $x$ and $y$ coordinate directions.

The simulation results for retrieved scattering parameters of the free metasurface are shown in the spectrum plot of Figure 3a. For the free metasurface in the absence of substrate or reflector the scattering parameters for forward/backward propagation are identical owing to symmetry. The effective properties calculations were made using a conventional retrieval process ${ }^{8}$ slightly modified as outlined in Swett ${ }^{6}$ for the selection of the phase complex root which considers the case made by Markel ${ }^{9}$ that material passivity fully constrains the imaginary part of permittivity to be positive, but does not preclude negativity in the imaginary part of permeability. Since the quantities of relative impedance and refractive index are not independent parameters but derived values from fundamental properties, the selection of one root fixes the value of the other ${ }^{10}$. Consequently, the retrieval process then need only ensure consistent use of the proper root throughout the properties calculations since these parameters are not fundamental quantities in Maxwell's equations but derived values. The selection here is based upon the complex phase root corresponding to positivity in the imaginary part of effective refractive index with the dependent parameter relative impedance. A pair of roots for each of the fundamental quantities, permittivity and permeability, is found with the proper
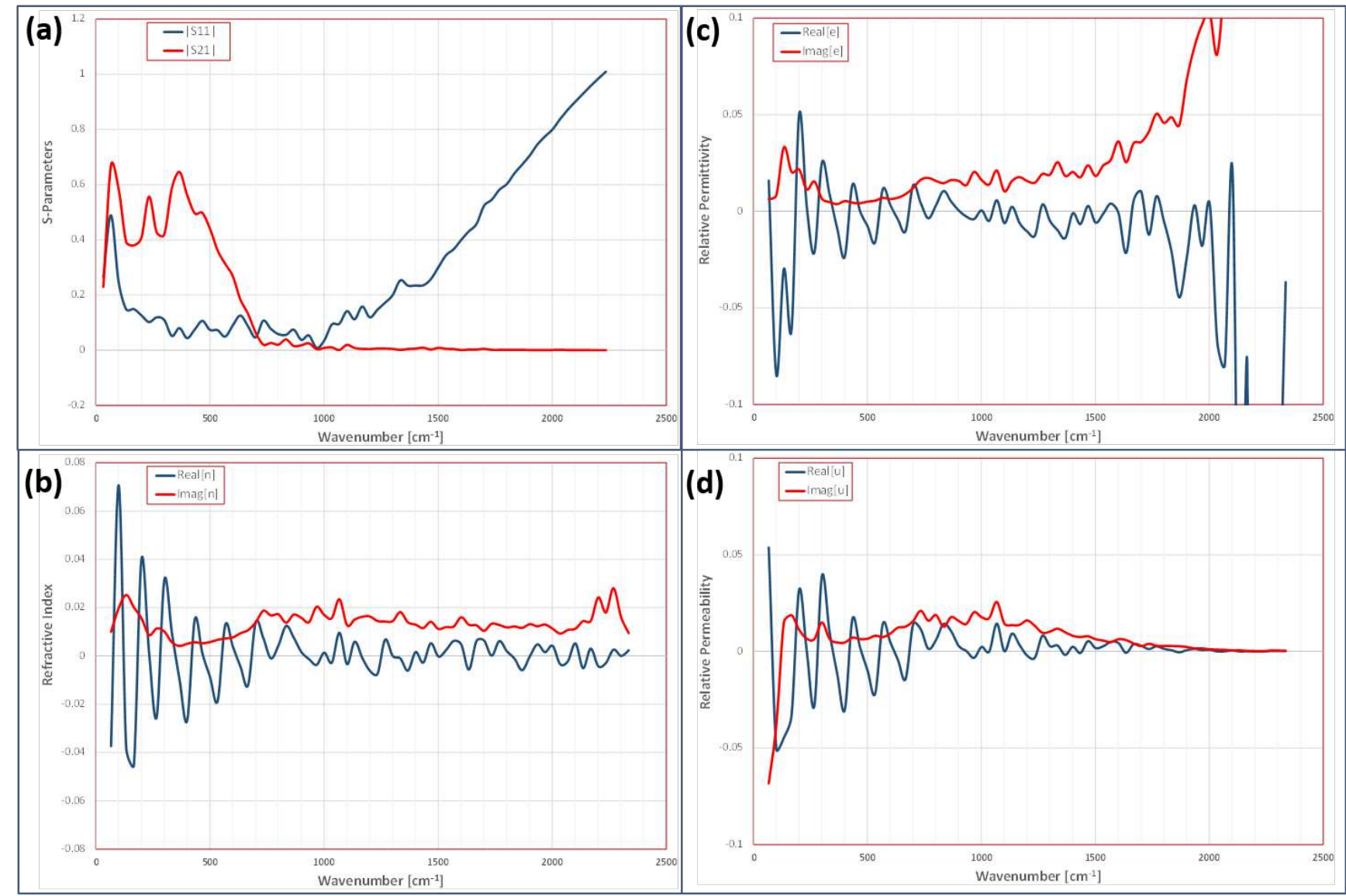

Figure 3. Metasurface scattering parameters retrieval simulations. (a) S-Parameters. (b) Effective refractive index. Dark blue line is real part and dark red line is imaginary part of index $n$. (c) Electrical permittivity, dark blue line is real part and dark red line is imaginary part of $\varepsilon$. (d) Magnetic permeability, dark blue line is real part and dark red line is imaginary part of $\mu$. 
selection being constrained by passivity to that which maintains positivity in the imaginary part of the effective permittivity. Then the only ambiguity in the retrieval process is cyclic branching in the real part of the phase parameter which can be addressed by generating the analysis from a sufficiently low frequency that ensures the retrieval slab thickness is thin compared to the incident wavelength and that the initial branch number in the analysis is strictly $m=0$. Any branching with increasing frequency can be found by comparison with multiple branch number spectra. In this analysis no branching from $m=0$ was found over the frequency range considered up to $2500 \mathrm{~cm}^{-1}$ with this inverted TanCirc mapping geometry.

The resulting refractive index spectra calculated for the inverted TanCirc geometry are shown in Figure $3 \mathrm{~b}$ with both real and imaginary components varying closely about zero over the wavenumber range of interest $\left(500-2000 \mathrm{~cm}^{-1}\right)$. The corresponding electrical permittivity and magnetic permeability spectra shown in Figure $3 \mathrm{c}$ and $3 \mathrm{~d}$ similarly indicate near zero effective electromagnetic properties with both real and imaginary parts varying closely to zero over the range of interest. For the free metasurface having no substrate or reflective ground plane the absorption spectrum can be calculated using:

$$
A=1-\left[\left|S_{11}\right|^{2}+\left|S_{21}\right|^{2}\right]
$$

The resulting absorption spectrum in Figure 4 for the free metasurface (no thermometric substrate or PEC ground plane) indicates a maximum absorption of $99.88 \%$ at $767 \mathrm{~cm}^{-1}(13 \mu \mathrm{m})$ with a $90 \%$ absorption bandwidth in the range between $600-$ $1534 \mathrm{~cm}^{-1}$ and $112 \%$ FWHM centered about $1218 \mathrm{~cm}^{-1}$. In this bandwidth the inverted TanCirc metasurface geometry is ultrathin at a small fraction $\lambda / 300$ of the center frequency incident wavelength. The imaginary components of the effective parameters spectra shown in Figure 3 indicate that the metasurface is a very low loss effective medium over this high absorption bandwidth, contrary to conventional understanding that intrinsically high loss media are necessary to develop high absorptivity in thin layers. Researchers have investigated the phenomena of perfect absorption in ultrathin metamaterial structures with near-zero-index that are coupled to either a PEC ground plane ${ }^{11}$ or on a metal substrate ${ }^{12}$. The phenomena of perfect absorption of the ultrathin metamaterial on a metal substrate was attributed to above-light-line surface plasmon polaritons that arise at the interface between the metasurface and the metal layer, inducing fast-wave non-radiative modes in the bilayer ${ }^{12}$, while the case coupled to a PEC ground plane was shown subsequently to be due to coherent cancellation, that is, interference in out-of-phase reflected waves between the metamaterial layer and the PEC substrate ${ }^{13}$. Neither coupling mechanism is directly applicable to the free metasurface absorption spectrum in Figure 4 having no PEC ground plane or substrate. The reasonable assumption is that the absorption phenomena found here for the inverted TanCirc metasurface geometry is related to surface

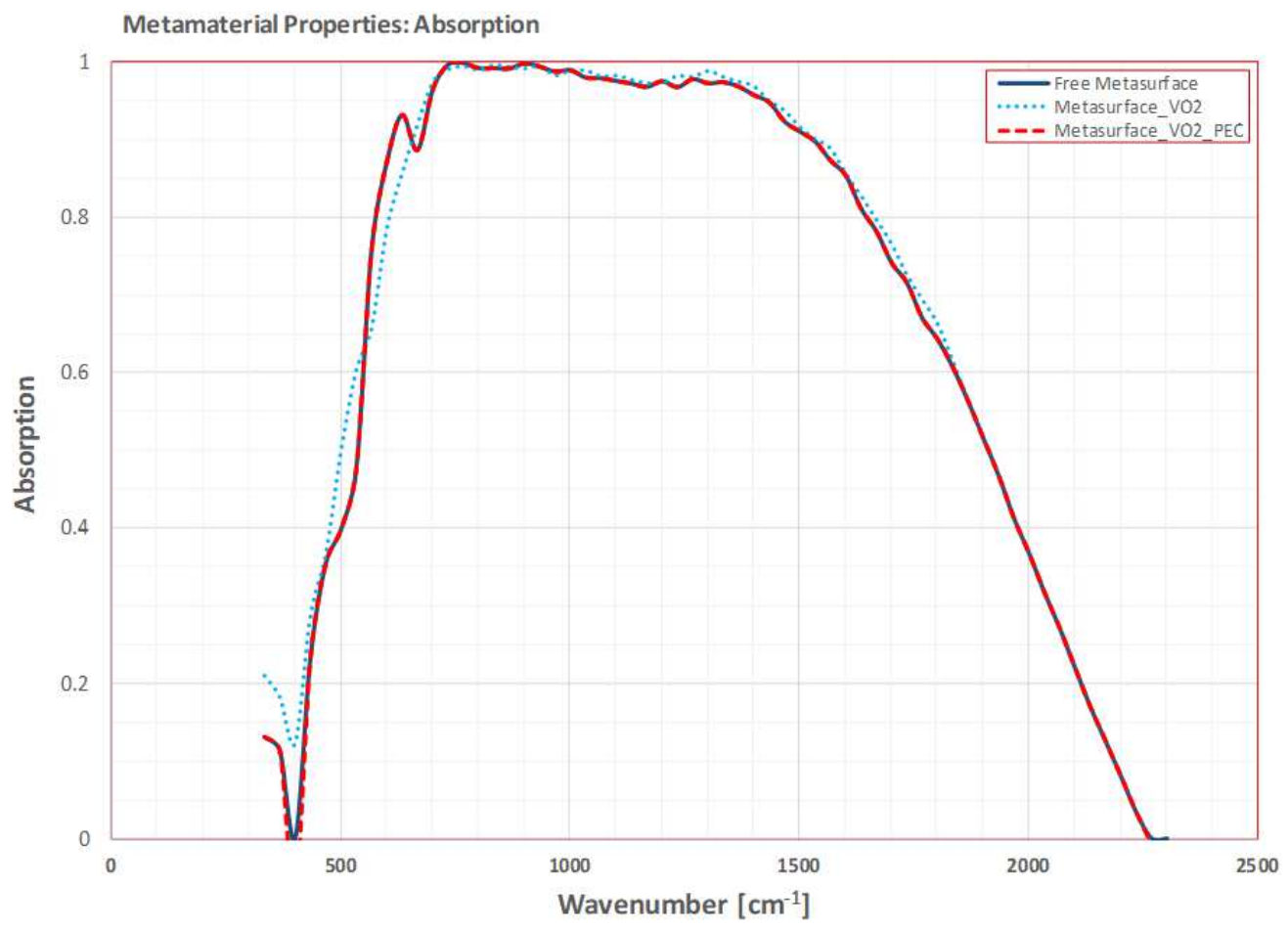

Figure 4. Absorption spectra, $30^{\circ}$ incidence. Solid dark blue line is free metasurface (no substrate/reflector). Dotted light blue line is metasurface on $135 \mathrm{~nm} \mathrm{VO} 2$ substrate. Dashed red line is metasurface on $135 \mathrm{~nm} \mathrm{VO} 2$ substrate and PEC reflector spaced $500 \mathrm{~nm}$ from substrate bottom surface. 
plasmon resonances and predominant losses developing from perhaps surface plasmon scattering ${ }^{14}$, the investigation of which is beyond the scope of this work. The absorption spectrum for the uncooled microbolometer design is superimposed with the free metasurface absorption in Figure 4, and shown to exhibit a predicted maximum absorption and bandwidth comparable to the free metasurface response. The electromagnetic response of the free metasurface for the perfect absorption resonance at 767 $\mathrm{cm}^{-1}$ is illustrated in the electric field lines of Figure 5a and magnetic field lines of Figure 5b. The loss mechanism for the free metasurface is only resistive and is illustrated in the current density plot of Figure $5 \mathrm{c}$ and the associated surface loss plot in Figure 5d. The regions of highest power dissipation appear to correspond generally to the central symmetry lines of each of the eight subcell geometric patterns.
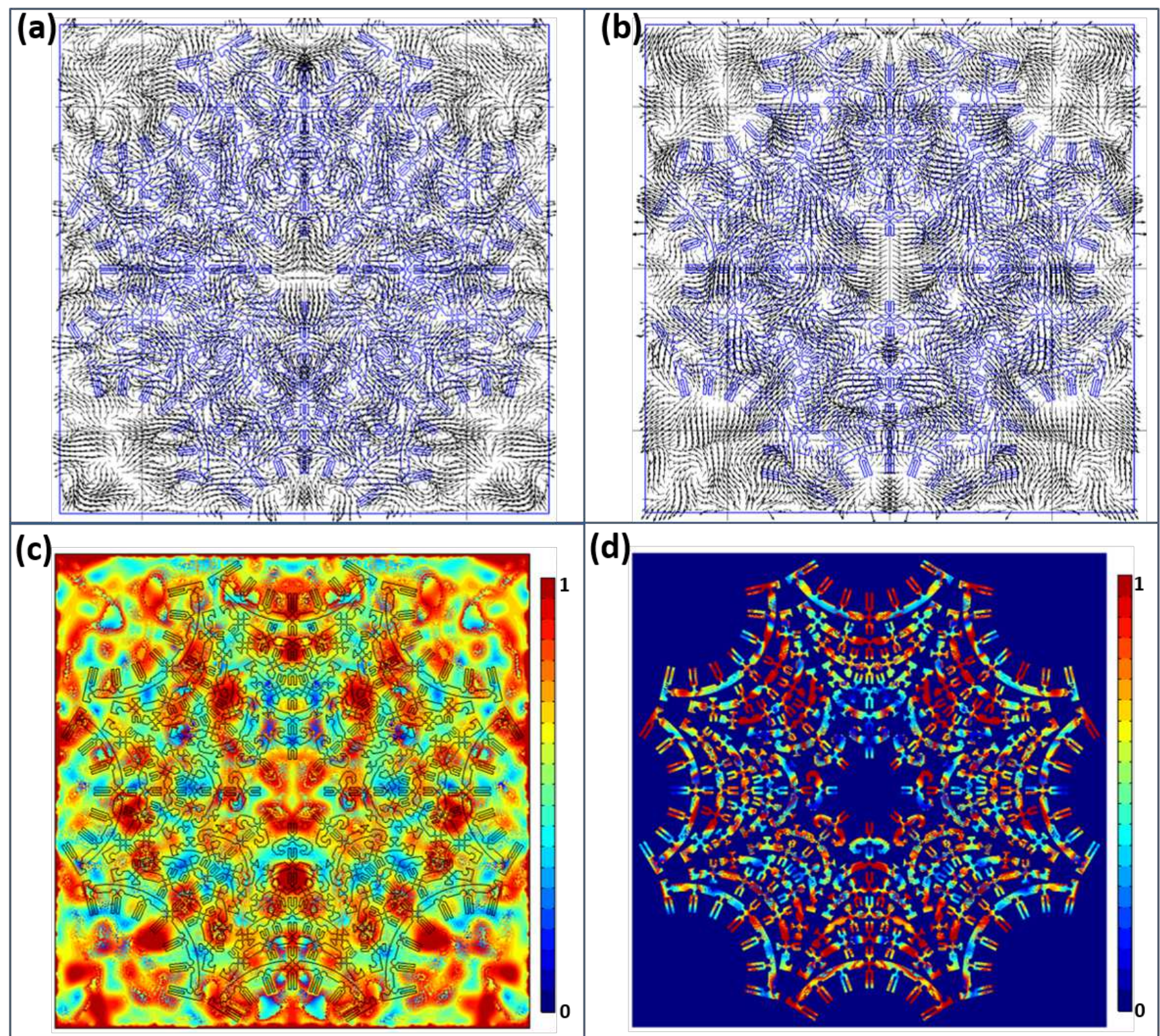

(d)

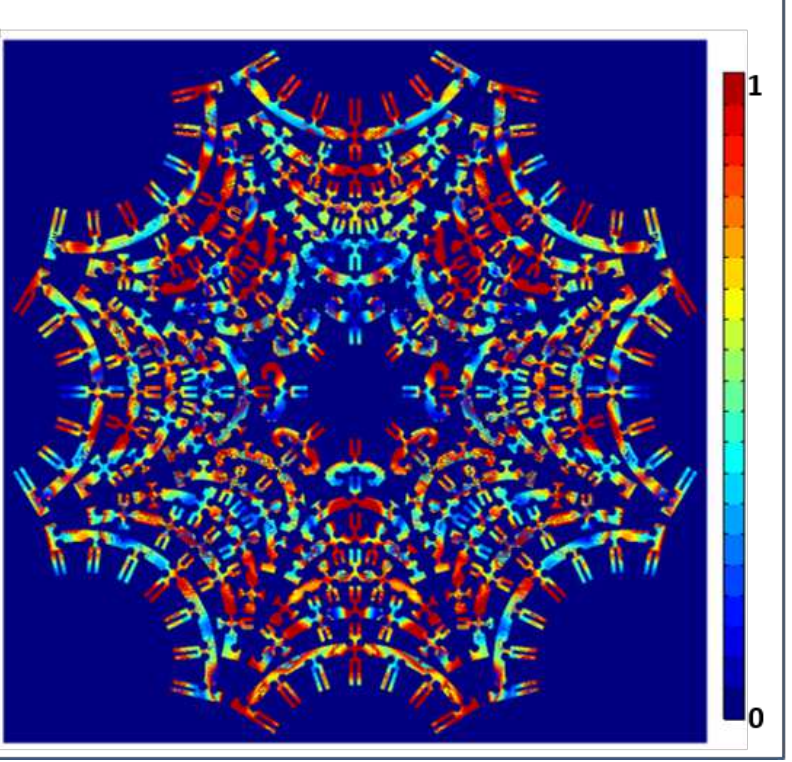

Figure 5. Electromagnetic response on the metasurface plane, $767 \mathrm{~cm}^{-1}$ wavenumber, (a) Electric field lines, (b) Magnetic field lines, (c) Current density (a.u.), (d) Surface loss (a.u.).

\section{Metasurface Uncooled Microbolometer}

The microbolometer detector design involves a micromachined bridge structure suspended over a readout integrated circuit (ROIC) substrate in which the Gold metasurface geometry is imprinted on a single thermometric layer of $\mathrm{VO}_{2}$ material, as illustrated in Figure 6. The design eliminates the typical support layers characteristic of conventional devices and is possible 

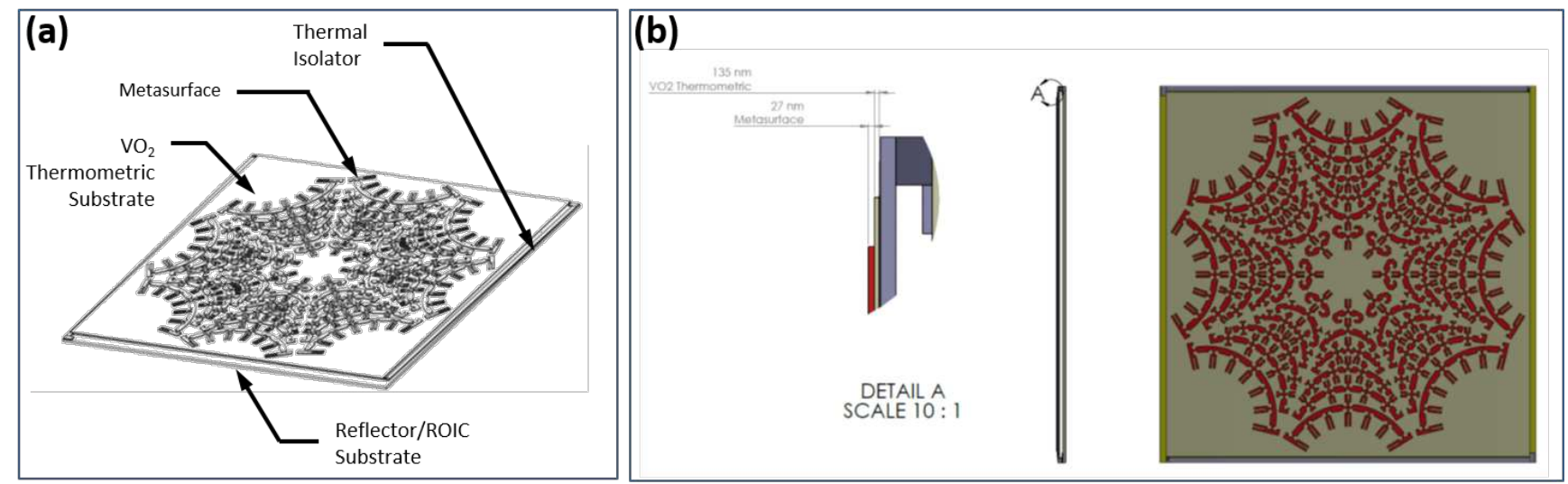

Figure 6. Uncooled microbolometer design concept, (a) Annotated isometric, (b) Detailed elevations.

due to the low mass loading from the ultrathin Gold metasurface layer. For a $135 \mathrm{~nm} \mathrm{VO}$ thermometric layer thickness and $27 \mathrm{~nm}$ Gold metasurface thickness with $35 \%$ areal fill factor, the resultant mass loading on the microbolometer develops a maximum bending stress of $7 \mathrm{kPa} / \mathrm{g}$ in the $\mathrm{VO}_{2}$ thermometric layer. The tensile strength of the $\mathrm{VO}_{2}$ material is $\sigma_{u l t}=172$ MPa translating into an ultimate shock capability $>24000 \mathrm{~g}$ 's, well in excess of the expected worst case shock loads that could be experienced downhole in a production logging environment $(<100 \mathrm{~g}$ 's). As a result, the single thermometric layer provides more than adequate structural support with this ultrathin metasurface for the expected downhole vibration and shock environments.
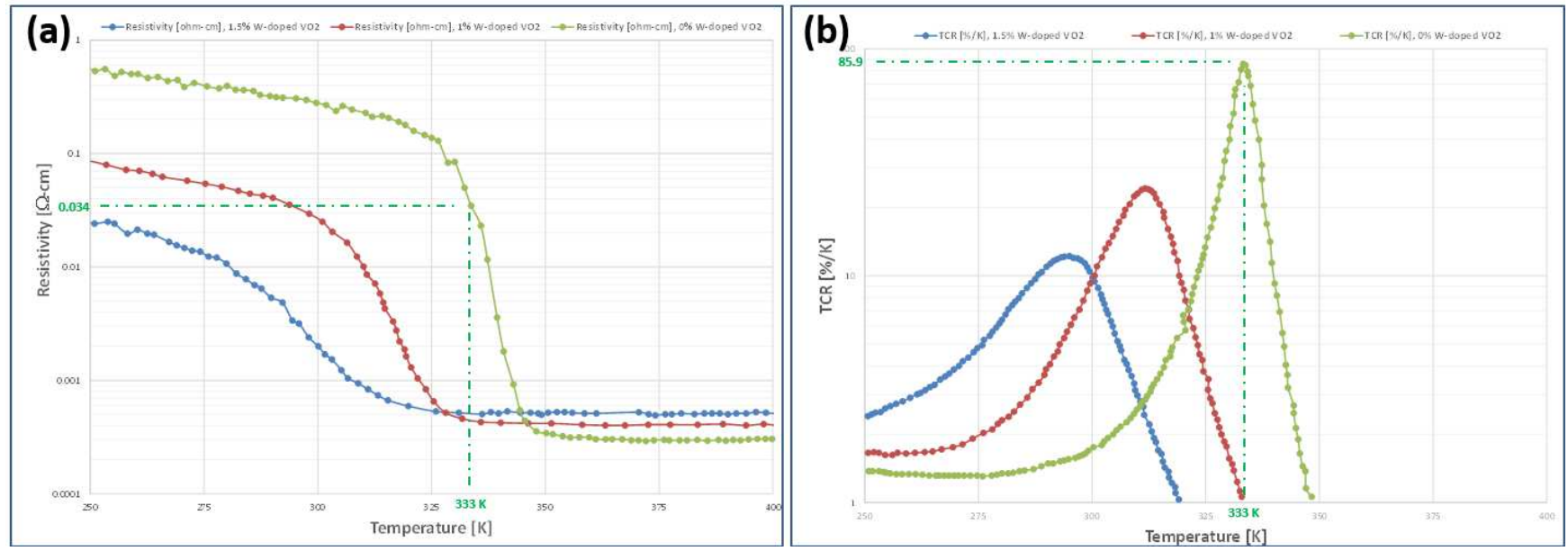

Figure 7. Doped and undoped $\mathrm{VO}_{2}$ properties: (a) Temperature dependence of electrical resistivity. (b) Temperature dependence of thermal coefficient of resistance (from Takami ${ }^{15}$ ).

The normalized detectivity is dependent upon the electrical resistivity and thermal coefficient of resistance of the thermometric $\mathrm{VO}_{2}$ layer, while the noise equivalent difference temperature (NEDT) is dependent upon its specific carrier density. The trends of the electrical resistivity and temperature coefficient of resistance properties of $\mathrm{VO}_{2}$ films with temperature were measured by Takami et. al ${ }^{15}$, and are shown in Figure 7, which clearly illustrates the metal-insulator-transition (MIT). For undoped $\mathrm{VO}_{2}$ film the data in Figure $7 \mathrm{a}$ indicates a resistivity at $60^{\circ} \mathrm{C}$ of approximately $3.5 \times 10^{-2} \Omega$-cm. The electron density of $\mathrm{VO}_{2}$ has been calculated based upon theoretical considerations by Pergament et. al ${ }^{17}$ as approximately $4 \times 10^{18} / \mathrm{cm}^{3}$. Using these material properties for the $\mathrm{VO}_{2}$ thermometric layer and the Comsol ${ }^{\circledR}$ MultiPhysics simulation results for the metasurface absorptivity, a microbolometer maximum detectivity $D^{*}$ of $1.5 \times 10^{10} \mathrm{~cm} \sqrt{\mathrm{Hz}} / \mathrm{W}$ at $333 \mathrm{~K}$, and NEDT of $1 \mathrm{mK}$ is calculated using the similar procedure as found in the literature ${ }^{6}$. The results are based upon $175 \mu \mathrm{A}$ bias current which creates a latent resistive temperature rise of $2.3 \mathrm{~K}$ in the microbolometer.

The direct comparison in Figure 8 includes the detectivity spectrum of the metasurface detector at $500 \mathrm{~Hz}$ modulation frequency superimposed onto the spectra for various commercially available infrared detector technologies operated at the 


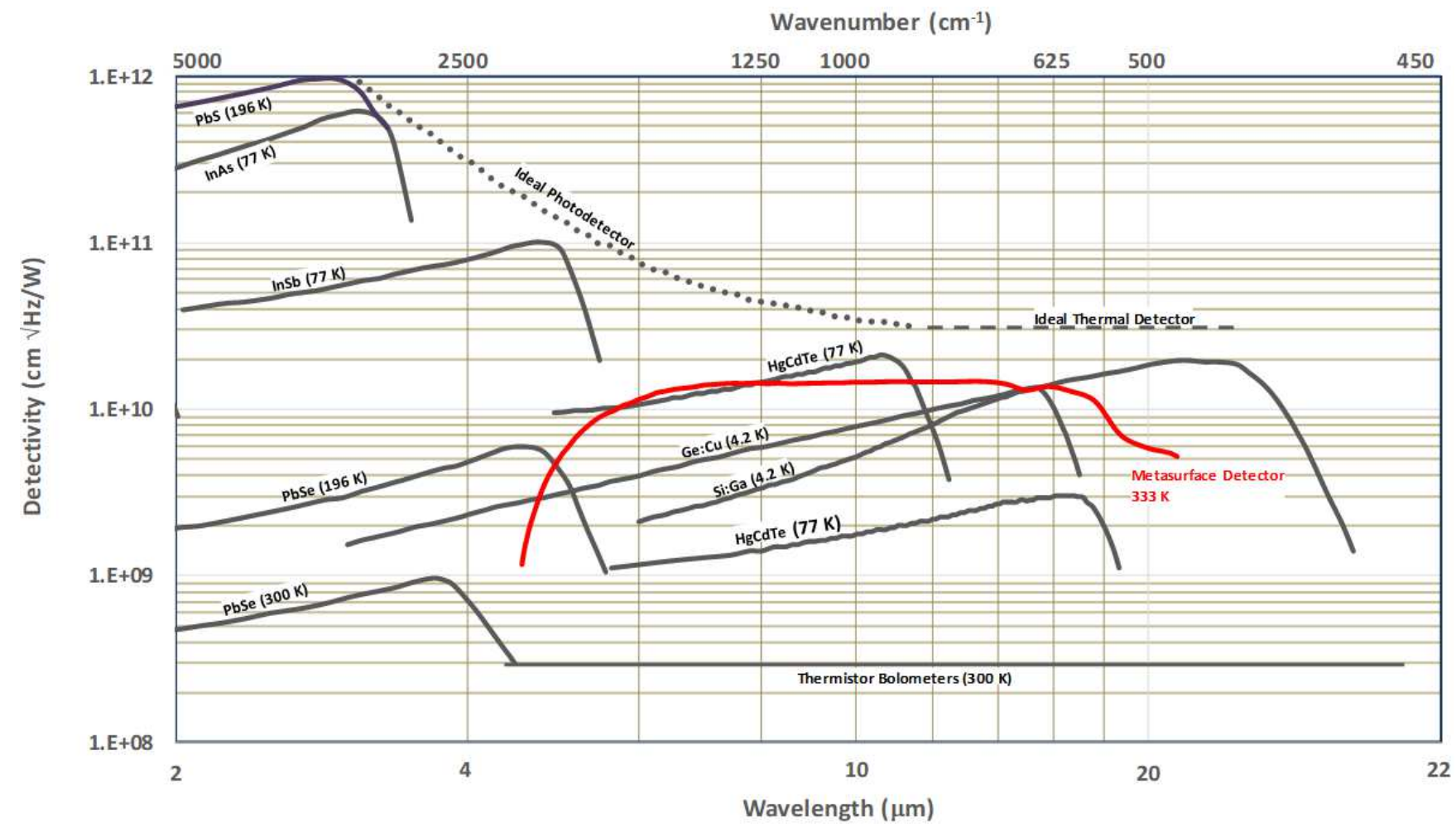

Figure 8. Comparison of spectral detectivity for the metasurface detector [RED], and various commercially available IR detectors (Hamamatsu Corp. ${ }^{16}$ ) operated at the noted temperatures. The modulation frequency for all detectors is $1000 \mathrm{~Hz}$, except for the state of the art uncooled thermistor bolometers at $10 \mathrm{~Hz}$ and the metasurface detector at $500 \mathrm{~Hz}$.

Table 1. Summary of metamaterial microbolometer design properties (Modulation $f_{m}=200 \mathrm{~Hz}$, Bandwidth $\Delta f=10 \mathrm{~Hz}$ ).

\begin{tabular}{|r|c|}
\hline Metasurface envelope & $Q 150 \times 0.027 \mu \mathrm{m}^{3}$ \\
\hline Maximum Absorption & $99.9 \%$ \\
\hline Modulation Frequency & $500 \mathrm{~Hz}$ \\
\hline $\mathrm{VO}_{2}$ thermometric substrate dimensions & $152 \times 152 \times 0.135 \mu \mathrm{m}^{3}$ \\
\hline $\mathrm{VO}_{2} \mathrm{TCR}, \beta$ & $0.8591 / \mathrm{K} @ 60^{\circ} \mathrm{C}$ \\
\hline $\mathrm{VO}_{2}$ Resistivity, $\mathrm{R}_{s}$ & $0.035 \Omega$-cm @ $60^{\circ} \mathrm{C}$ \\
\hline Ri electrode dimensions $(4)$ & $1 \times 0.5 \times 150 \mu \mathrm{m}^{3}$ \\
\hline Besistance, $R$ & $2570 \Omega$ \\
\hline Bias Current, $I_{b}$ & $175 \mu \mathrm{A}$ \\
\hline Resistive Temperature Rise, $\Delta T_{I_{b}}$ & $2.3 \mathrm{~K}$ \\
\hline Thermal Conductance, $G_{t h}$ & $3.0 \times 10^{-7} \mathrm{~W} / \mathrm{K}$ \\
\hline Thermal Capacitance, $C_{t h}$ & $9.74 \times 10^{-9} \mathrm{~J} / \mathrm{K}$ \\
\hline Thermal Time Constant, $\tau_{t h}$ & $32.5 \mathrm{~ms}$ \\
\hline $\mathrm{NEDT}$ & $1 \mathrm{mK}$ \\
\hline Maximum Responsivity, $R_{v}$ & $11.3 \mathrm{kV} / \mathrm{W}$ \\
\hline Maximum Detectivity, $D^{*}$ & $1.5 \times 10^{10} \mathrm{~cm} \sqrt{\mathrm{Hz}} / \mathrm{W}$ \\
\hline
\end{tabular}

noted temperatures and over the wavenumber range from $5000-250 \mathrm{~cm}^{-1}$. The superimposed metasurface microbolometer spectrum indicates a detectivity comparable to the performance conventionally associated with state of the art cryogenically cooled detectors. Consequently, this level of detection would represent a disruptive technology for uncooled microbolometers in consideration of the comparison with such as a photon detection based $\mathrm{Ge}: \mathrm{Cu}$ cooled to $4.2 \mathrm{~K}$. The details of the metasurface detector design are summarized in Table 1. 

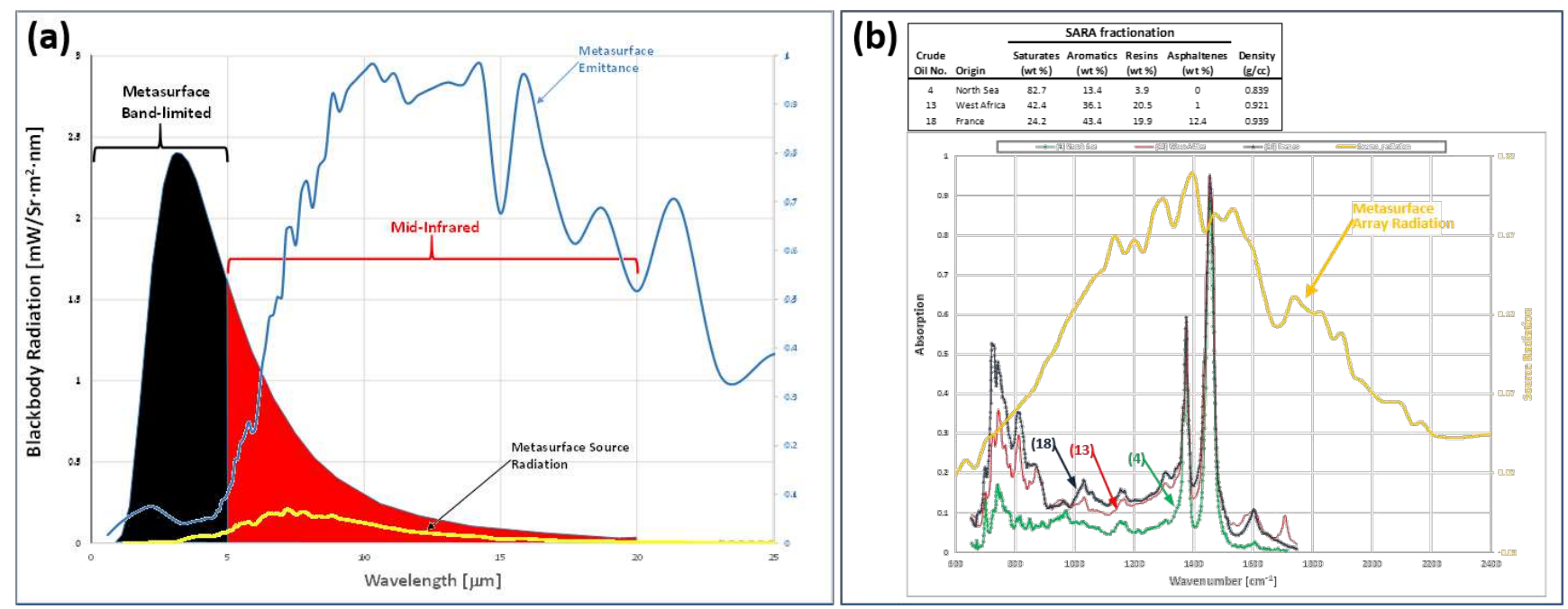

Figure 9. Metasurface source radiation. (a) Illustration of the intrinsic band-limiting from the metasurface array at $900 \mathrm{~K}$, normal exidence. (b) Superposition of metasurface array radiation on absorption spectra for three crude oils from different global reservoirs ${ }^{18}$. The SARA fractions for the crude oil samples are summarized in the upper left table.

\section{Metasurface Thermal Infrared Source}

The principle on which conventional infrared sources operate is based upon emittance over the classical Blackbody radiation spectrum. The radiation spectrum from these type emitters is generally concentrated in the visible and near infrared wavelength ranges. For mid-infrared spectroscopy applications in downhole chemical analysis, we are interested in the electromagnetic wavelength range $5-20 \mu \mathrm{m}\left(2000-500 \mathrm{~cm}^{-1}\right)$ so that the predominant portion of the radiation from these type sources lies outside this bandwidth below $5 \mu \mathrm{m}$ wavelength and would represent a heat load limiting the downhole integration of such devices. In illustration, consider the Blackbody radiation spectrum shown in Figure 9a corresponding to a radiating blackbody at $900 \mathrm{~K}$. The spectrum shows that the energy radiated in the wavelength range below $5 \mu \mathrm{m}\left(2000 \mathrm{~cm}^{-1}\right)$ is significant in relation to the energy in the wavelength range beyond $5 \mu \mathrm{m}$, in fact comprising almost $60 \%$ of the total radiation. This is one of the practical obstacles confronting MEMS scale miniaturization of laboratory quality spectroscopy instruments downhole, as thermal management options are severely limited in logging tools due to the extreme wellbore temperatures and miniature volumetric constraints. Here we leverage the unusual properties of the inverted TanCirc metasurface perfect absorber to create a bandwidth-limited infrared source that circumvents this problem. From Kirchoff's law we understand that, for an arbitrary body emitting and absorbing thermal radiation in thermodynamic equilibrium, the absorptivity and emissivity are equivalent. Thus, the bandwidth-limited perfect absorptivity characteristics of the metasurface translate into bandwidth-limited perfect emissivity characteristics as well. This concept is illustrated in the plot of the Figure 9a depicting a comparison of the emissivity of the copper metasurface cells imprinted onto a $\mathrm{Si}_{3} \mathrm{~N}_{4}$ substrate controlled to $900 \mathrm{~K}$ and the natural Blackbody radiation spectrum at the same temperature. The comparison in the figure indicates that the bulk of the waste heat problem is eliminated by the bandwidth-limited emissivity of the metasurface array, with the electromagnetic radiation concentrated within the wavenumber range $2000-500 \mathrm{~cm}^{-1}(5-20 \mu \mathrm{m})$ being of specific interest for the downhole chemical analysis application as highlighted in the crude oil absorption spectra shown in the right-side of Figure 9b. The emissivity characteristics for the metasurface thermal source were determined using an electromagnetic simulation model similar to the previous one described for absorption properties of the metasurface microbolometer. The metasurface Copper material is modeled with an electrical permittivity that is frequency dependent following a Drude model $^{7}$ with a simple hyperbolic temperature dependence estimate of the form:

$$
\varepsilon_{c u}(\omega)=\left[1-\frac{\omega_{p}^{2}}{\omega^{2}+\gamma^{2}}\right]+j\left[\left(\frac{\omega_{p}^{2} \gamma}{\omega^{3}+\omega \gamma^{2}}\right) /\left(1+\alpha\left(T-T_{0}\right)\right)\right]
$$

where $\omega_{p}=2.118 \times 10^{15} \mathrm{~Hz}$ is the plasma frequency, $\gamma=23.09 \times 10^{12} \mathrm{~Hz}$ is the electromagnetic damping frequency, and $\alpha=4.29 \times 10^{-3} 1 /{ }^{\circ} \mathrm{C}$ is the Copper resistivity temperature coefficient with respect to ambient conditions at temperature $T_{0}$. For the emittance analysis, the metasurface pattern is modeled as an imprint on $500 \mathrm{~nm} \mathrm{Si}_{3} \mathrm{~N}_{4}$ with the back surface of the substrate modeled as a PEC boundary condition, the laminate suspended in the vacuum waveguide.

The intrinsic bandwidth limited radiation of the metasurface infrared source is coincident with important chemical spectra of downhole hydrocarbons as shown in the spectra overlay plot of Figure $9 \mathrm{~b}$ from experimental data by Aske et. $\mathrm{al}^{18}$. The 


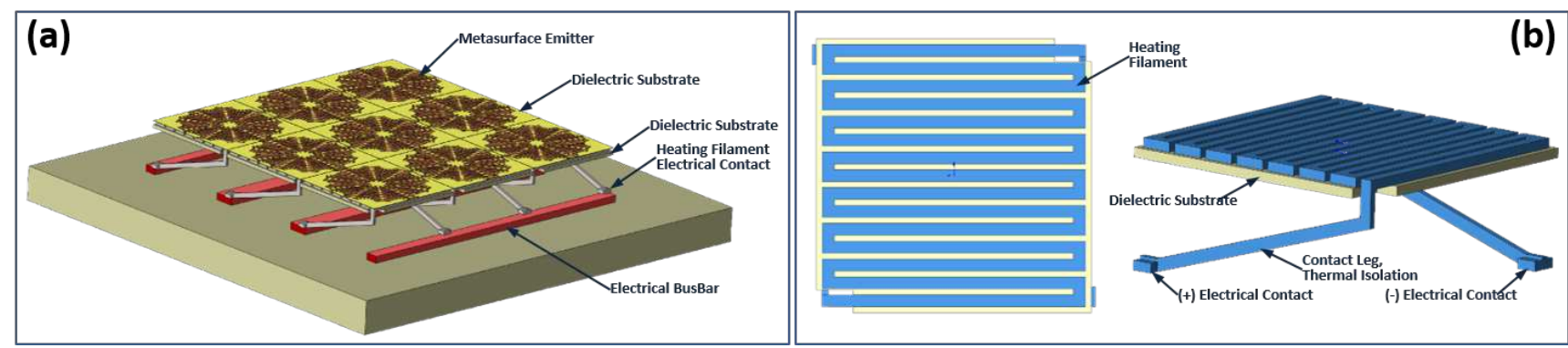

Figure 10. Metasurface infrared source. (a) Metasurface array assembly, (b) Substrate resistive heat generation.

spectra in Figure 9b show the differences between crudes of different SARA fractions, and emphasize the high distinctions that exist in the lower wavenumber range below $1400 \mathrm{~cm}^{-1}$ in which the metasurface absorptivity remains generally above $95 \%$. The metasurface array infrared source module is comprised of (i) a metasurface radiation source, (ii) an off-axis-parabolic (OAP) folding mirror, and (iii) an optical-tube which houses the two optical components and creates the knife-edge baffle diameter of the incidence beam onto the OAP mirror-1. The details of the metasurface infrared source array are illustrated in the left side of Figure 10 and the details of the emitter heating filament design are illustrated in the right side of Figure 10. The target temperature of the source array is maintained by controlling the electrical current in the heating filament through a pulse-width-modulation scheme on the electrical voltage across the resistive filament. The array support functions as thermal isolation with a nominal leakage of $690 \mathrm{~mW}$ at $900 \mathrm{~K}$ to the optical bench with temperature controlled to $333 \mathrm{~K}\left(60{ }^{\circ} \mathrm{C}\right)$. This leakage can be directly absorbed using a $1 \times 2$ array of a commercial off-the-shelf Peltier device capable of maintaining a bi-directional $40{ }^{\circ} \mathrm{C}$ temperature differential under $0.8 \mathrm{~W}$ heat load, using $1.5 \mathrm{~W}$ supply power. For downhole wireline logging operations this is a minimal power requirement, and $40^{\circ} \mathrm{C}$ bi-directional temperature differential is compatible with the target application involving environmental conditions of $40{ }^{\circ} \mathrm{C}-100{ }^{\circ} \mathrm{C}$ with an optical bench control temperature of $60{ }^{\circ} \mathrm{C}$.

\section{Downhole FT-MIR Spectroscopic Probe}

Decreasing the size of existing laboratory grade FT-MIR spectroscopic instruments is one of the major prerequisites to migrating this technology to remote sensing applications such as downhole production logging systems. For downhole applications an equally critical criterion for adaptation is high-temperature capability, which is in direct conflict with many of the fundamental technologies used in conventional mid-infrared spectrometery devices. To address the problem of migrating standard laboratory grade mid-infrared spectrometry downhole, the current state of the art holds several main challenges to downhole application, (i) development of laboratory grade spectroscopy at elevated temperature without the need for cryogenically cooled detector technologies, (ii) miniaturization of the infrared source and interferometer assembly down to a scale amenable with integration into downhole logging instrument platforms, and finally (iii) maintenance of sufficiently fine spectral resolution $\Delta \lambda$ in the miniaturization to facilitate discrimination between the variety of chemicals that may be encountered downhole in wellbore fluids. The most daunting of these challenges is certainly the goal to achieve laboratory quality MIR spectroscopy at downhole elevated temperatures in an uncooled detector technology due to the fact that current technologies have limited detectivity in the longer wavelength regime unless enhanced with active cooling.

In laboratory grade benchtop mid-infrared spectrometers, one of the more common design approaches is the use of a Michelson type interferometer combined with Fourier transform postprocessing. The operation of this type spectroscope is based upon separating an incident beam of radiation into two beams by means of a beamsplitter, whereupon a path length difference between the separated beams is introduced by antisymmetric movement of both of two reflecting elements. This path length difference creates an interference between the recombined beams at the beamsplitter, resulting in a change in the intensity of the output beam as a function of the relative path length difference. This interference mechanism is called an interferometer. The intensity of the interferogram can be monitored as a function of path difference (i.e., relative displacement between the reflecting elements) using an appropriate detector. Fourier transformation techniques can then be applied to the raw interferogram data to convert the spectra from the relative displacement domain to a radiation wavelength domain, and this can then be used to analyze the absorption spectra characteristics and subsequently the chemical composition of the sample. We use a similar design approach here in developing a miniaturized downhole spectroscopic probe of the Michelson-type.

A set of graphical views for the downhole FT-MIR spectroscopic probe design is shown in Figure 11. The probe assembly includes the miniaturized optical train and MEMS based Michelson-type interferometer, the ATR prism for fluid sample sensing, a flow-through shroud to protect the prism sample interface from potential large debris impact, pressure housing, and pressure feedthrough multi-pin connector. The probe pressure housing outer diameter is $11.95 \mathrm{~mm}$ which is compatible with integration 

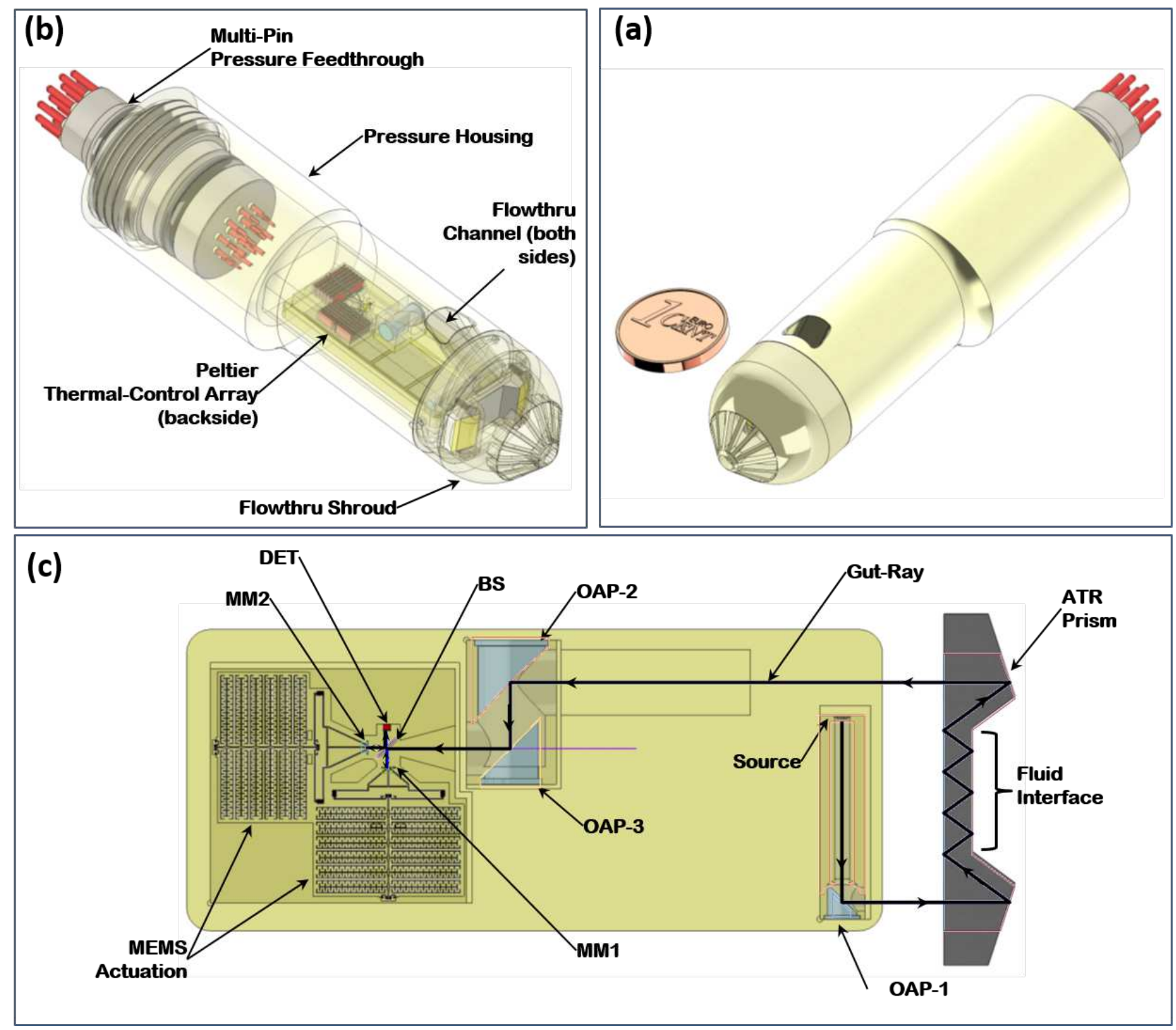

Figure 11. Downhole FT-MIR Probe: (a) Relative scale isometric view, (b) Transparent isometric view, (c) Optical train components and Gut-Ray trace.

into a sensor array type configuration for highly deviated and lateral wellbore logging applications.

The optical train is designed to confine the beam path within a $2 \mathrm{~cm}$ probe diameter as well as recover the majority of the emitted source intensity onto the detector. The miniaturized optical train design is based on integrating the uncooled metasurface microbolometer with a MEMS based interferometer, Ge ATR optical prism, and metasurface thermal infrared source. The thermal infrared source is comprised of a $3 \times 3$ array of metasurface cells each $150 \mu \mathrm{m}$ in diameter. The source radiation is directed along an optically black tube with the output aperture onto the first off-axis-parabolic (OAP) mirror being defined by a single knife-edge baffle. The ATR prism receives the folded OAP-1 beam bundle and uses a stepped thickness to produce three (3) reflections of an approximately $\varnothing 1.4 \mathrm{~mm}$ over a $5 \mathrm{~mm}$ length fluid sampling interface, with the goal to constrain the total ATR prism length to less than $15 \mathrm{~mm}$. The ATR prism output beam is directed to a subsequent two-mirror fold-focus/fold-focus assembly (OAP-2 \& OAP-3) then output onto the ZnSe 50/50 beamsplitter. Here the beam divides into two orthogonal paths of equal transmission: path-1 is created by a $50 \%$ reflection onto the moveable mirror MM1 and path- 2 is created by a 50\% transmission onto moveable mirror MM2. Each moveable mirror reflects the individual beams back onto the beamsplitter, with the path-1 having a 50\% transmission and the path-2 having a $50 \%$ reflection finally onto the metasurface detector. The uncooled microbolometer detector is comprised of a $2 \times 2$ array of metasurface cells of the same geometry as the 


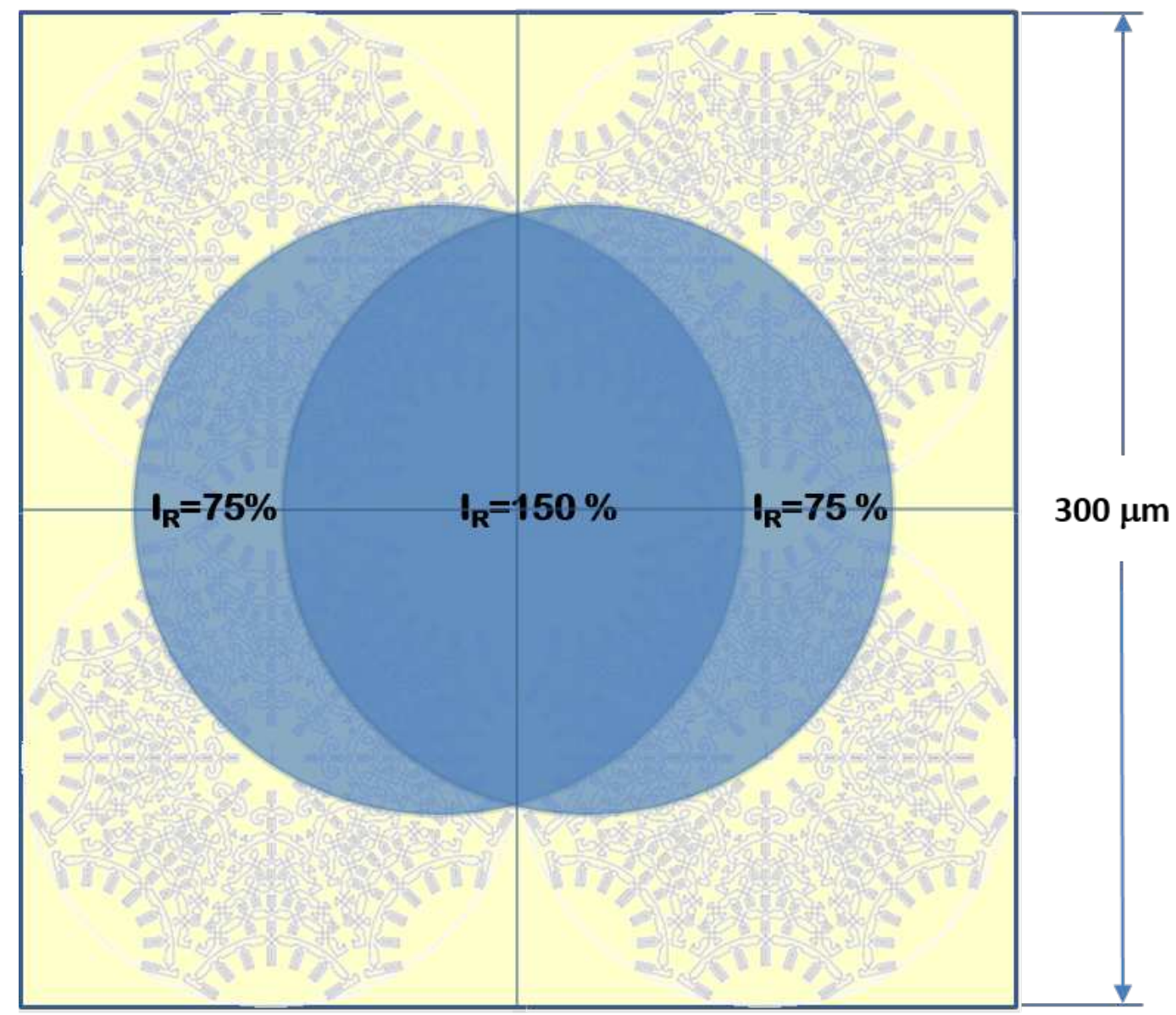

Figure 12. FT-MIR metasurface detector incidence pattern. The $2 \times 2$ metasurface array imprint is shown in background.

thermal infrared source cells. The two beams recombine on the metasurface detector with a footprint formed by two eccentric circles as illustrated in Figure 12, fitting within an oval-shaped envelope $0.184 \mathrm{~mm} \times 0.228 \mathrm{~mm}$. Due to the eccentricity of the two beams the detector spot pattern is comprised of a non-uniform intensity central double-crescent with $150 \%$ net intensity signal and two single-crescent side-lobes with $75 \%$ net intensity each. The central double-crescent and the two single-crescent side-lobes have approximately equal areas. This results in about $54 \%$ of the detector spot pattern area having $150 \%$ net relative intensity $\left(\sim 1000 \mathrm{~mW} / \mathrm{cm}^{2}\right)$, and the remainder comprised in the side-lobes with $75 \%$ net relative intensity. A graphical summary of the spectrometer design is illustrated in Figure 13a for a set of mid-plane emittance points on the infrared metasurface source, with the gut-ray coordinates, beam diameter variation, and intensity throughput for the two moveable mirror paths shown in the tabular listing in Figure 13b.

The MEMS interferometer utilizes a pair of on-chip electrostatic actuators that drive movable micro-mirrors through a displacement amplification mechanism. The conventional obstacle to sufficient spectral resolution for downhole chemical discrimination in a MEMS device derives from the fundamental limit in interferometric spectroscopy whereby the resolution $\Delta \lambda$ is inversely proportional to the amplitude of the interferometer mirrors motion. The conventional spectral resolution limitations encountered in interferometric miniaturization are overcome in this invention by a displacement amplification mechanism with about 9.4:1 translation of actuator motion to the moveable mirror element. The interferometer is comprised of an input beam fiber delivered from a conventional ATR window that allows interface to the wellbore fluids, two moveable mirror assemblies, a 50/50 infrared beamsplitter, and the metasurface detector array. A graphical illustration of the MEMS interferometer mechanism is shown in the plan view of Figure 14a and the isometric view of Figure 14b .

Each moveable mirror is controlled by an individual electrostatic comb-drive actuator and displacement amplification mechanism as illustrated in Figure 14c. The displacement amplification mechanism is created through the combination of a symmetric fulcrum about the actuator axis and three serpentine geometry moment release flexures. The comb-drive actuator imparts motion $\Delta_{\text {Act }}$ on the actuator central axis which activates the fulcrum lever about the constraint points 'F' resulting in an amplification of this motion at the moveable mirror. The three pairs of serpentine flexures are designed to function 


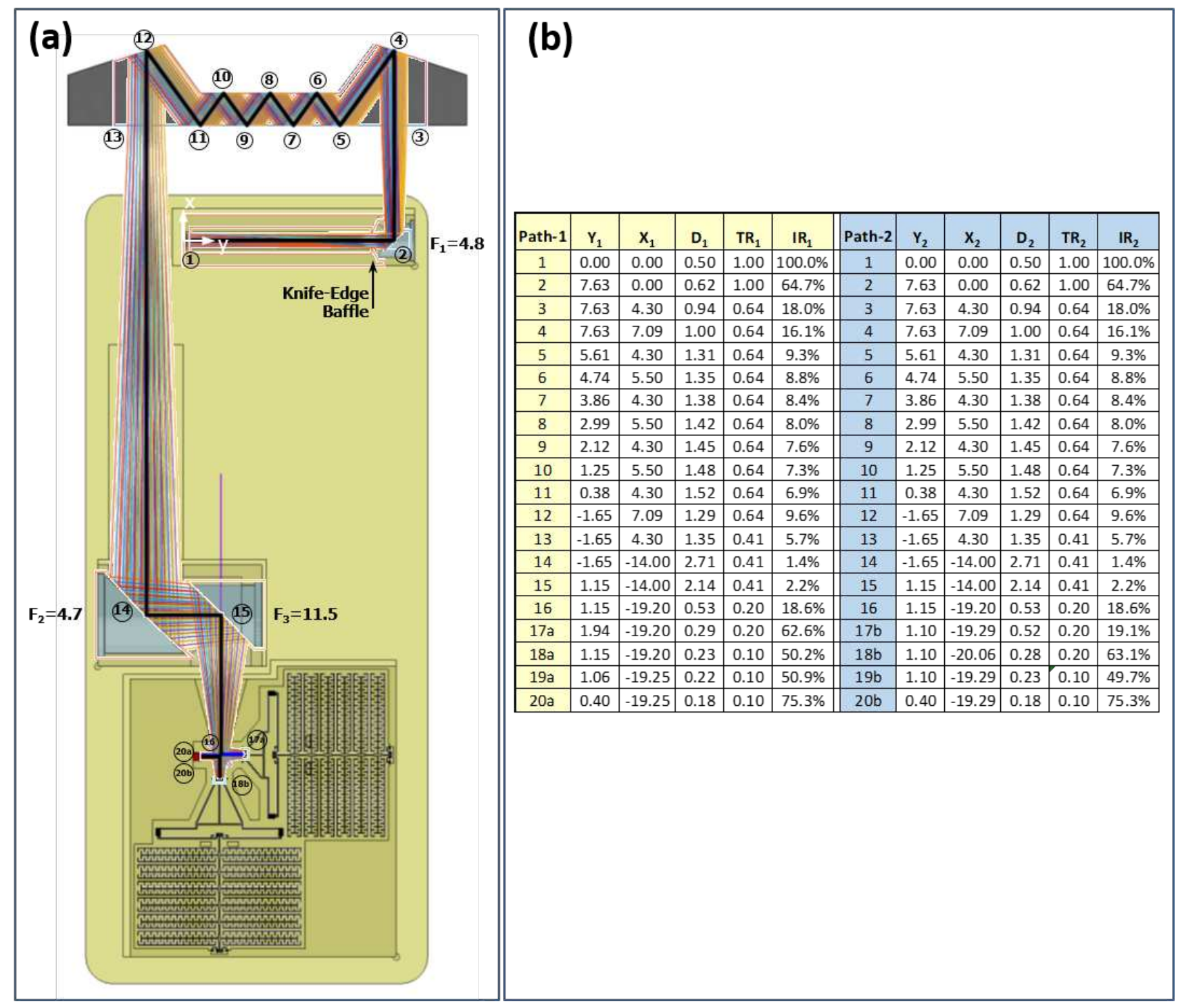

Figure 13. Optical train 2-D simulation. (a) Beam propagation and divergence/convergence, (b) Gut-ray coordinates $\left(X_{n}, Y_{n}\right)$, beam diameter $\left(D_{n}\right)$, transmission $\left(T_{n}\right)$, and relative intensity $\left(\mathrm{IR}_{n}\right)$ changes through optical train, units in millimeters.

as quasi-perfect hinge joints at each location. The degree of departure from the perfect hinge moment release degrades the mirror/actuator amplification ratio. For the idealized case in which the three pairs of flexures could be replaced by perfect ball-joints, the amplification ratio would be approximately 10:1 whereas in the practical design case involving the quasi-perfect serpentine flexures the amplification ratio is 9.4:1 due to the incomplete release of the moment. To increase the voltage limit at which the comb-drive experiences lateral instability, a sway stabilization flexure mechanism is integrated at the extreme location from the amplification mechanism. A graphical illustration of the moveable mirror motion due to the amplification mechanism is shown in Figure 14d. The illustration in Figure 14d indicates the electrostatic actuator displacement of 27 $\mu \mathrm{m}$ is increased to $256 \mu \mathrm{m}$ by the action of the amplification mechanism. This amplification of the electrostatic actuator displacement at the two moveable mirrors creates an interferometric spectral resolution of $10 \mathrm{~cm}^{-1}$ over the mid-IR spectral range up to $2000 \mathrm{~cm}^{-1}$. The maximum quasi-static stress developed in the moveable assembly flexures during actuation is approximately $350 \mathrm{MPa}$, well within the single-crystal Silicon material infinite life fatigue strength ${ }^{19}$. The moveable assembly has a fundamental resonance frequency mode at $198 \mathrm{~Hz}$ in the actuation direction, and $465 \mathrm{~Hz}$ in the lateral direction. These fundamental resonance frequencies lie within the major downhole random vibration spectra that is concentrated below $1 \mathrm{kHz}$, and will be susceptible to excitation. The lateral response is of critical interest due to the potential that vibrational excursions violate the minimum finger gap for lateral voltage stability. For a $110 \mu \mathrm{m}$ finger gap and $\pm 550 \mathrm{~V}$ maximum potential, the 

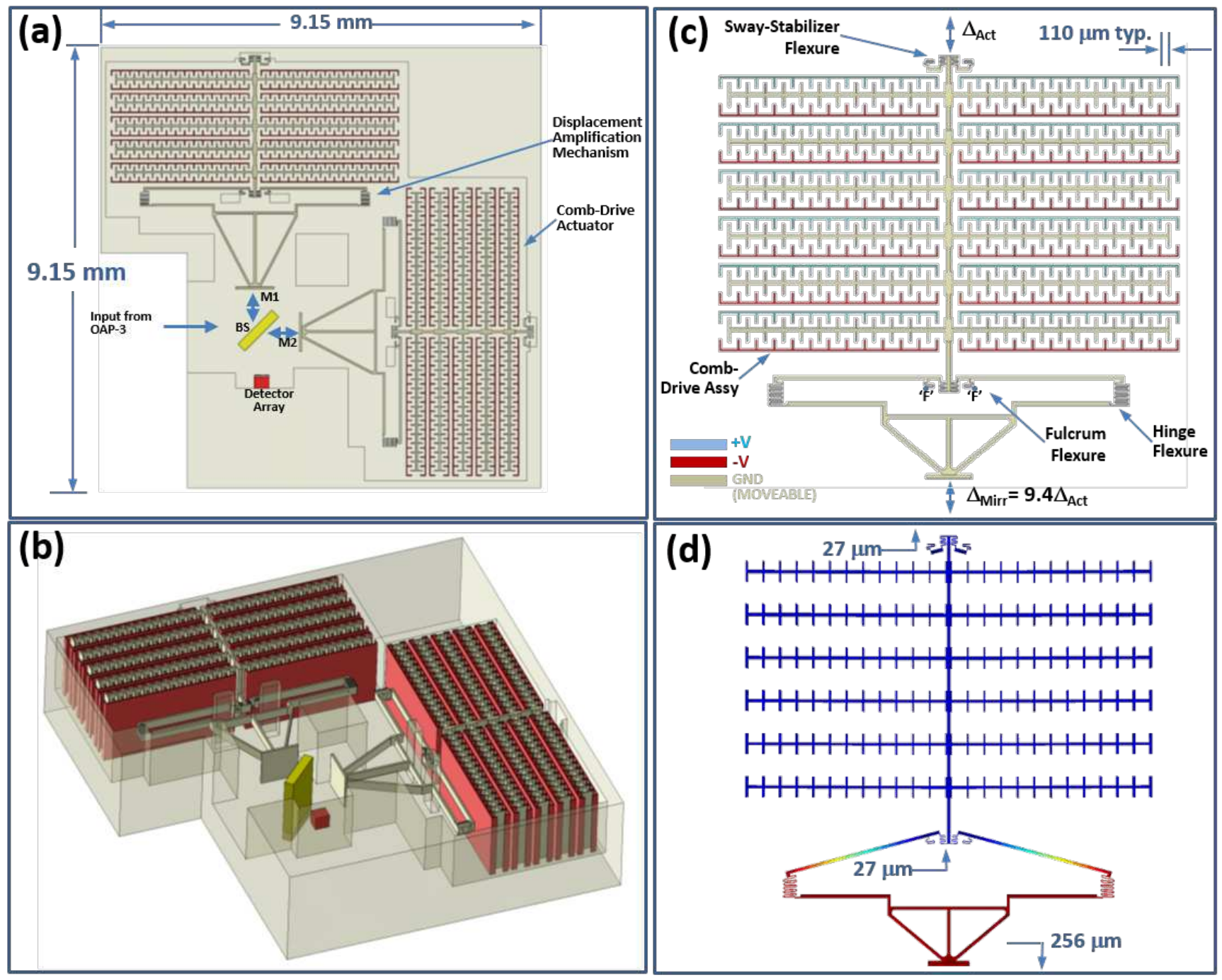

Figure 14. MEMS Interferometer, (a) Plan View, (b) Isometric View, (c) Comb-drive actuator and displacement amplification mechanism layout, (d) Actuator amplification mechanism, moveable mirror motion.

allowable lateral dynamic displacement is $\pm 17 \mu \mathrm{m}$, indicating a $15 \mathrm{Grms}$ effective dynamic load capacity which exceeds wireline random vibration requirements typically less than $10 \mathrm{Grms}$. The moveable mirror scan frequency is $2.4 \mathrm{~Hz}$ and cannot act as an excitation source for the moveable assembly at $198 \mathrm{~Hz}$ resonance frequency.

For in situ downhole chemical analysis applications, a sample rate on the order of once every second or so is minimally acceptable. For the $10 \mathrm{~cm}^{-1}$ resolution achievable with the described MEMS FT-MIR interferometer and a modulation frequency of $500 \mathrm{~Hz}$, a $2.4 \mathrm{~Hz}$ sample rate is achievable using one interferogram sweep per sample measurement over the spectral range up to $2000 \mathrm{~cm}^{-1}$. This limited number of interferogram sweeps/sample should provide an acceptable spectroscopy measurement for the downhole logging applications intended owing to the intrinsic low noise characteristics of the microbolometer evidenced in $1 \mathrm{mK}$ NEDT.

The rate at which sampling is made affects the resolution of the downhole analysis logging operation, but the accuracy is substantially dependent upon the correlation of the fluid properties on the ATR sampling interface with the actual properties of the fluid surrounding the probe. The correlation quality is controlled by the sampling cavity design which involves diversion and flowthrough of the flow stream incident on the probe with the rapidity at which the fluid in the ATR sampling cavity exchanges being the design basis for the cavity flowthrough geometry. Wellbore flow streams may contain solids and particles that pose the risk of potential damage to the ATR prism sampling surface and must be filtered to reject particles larger than approximately $\varnothing 1.5 \mathrm{~mm}$ using a protective shroud forming a cavity requiring a flowthrough design as illustrated in the plan and elevation views of Figure 15a. The lag time between fluid transition on the ATR sampling interface and that occurring in the 

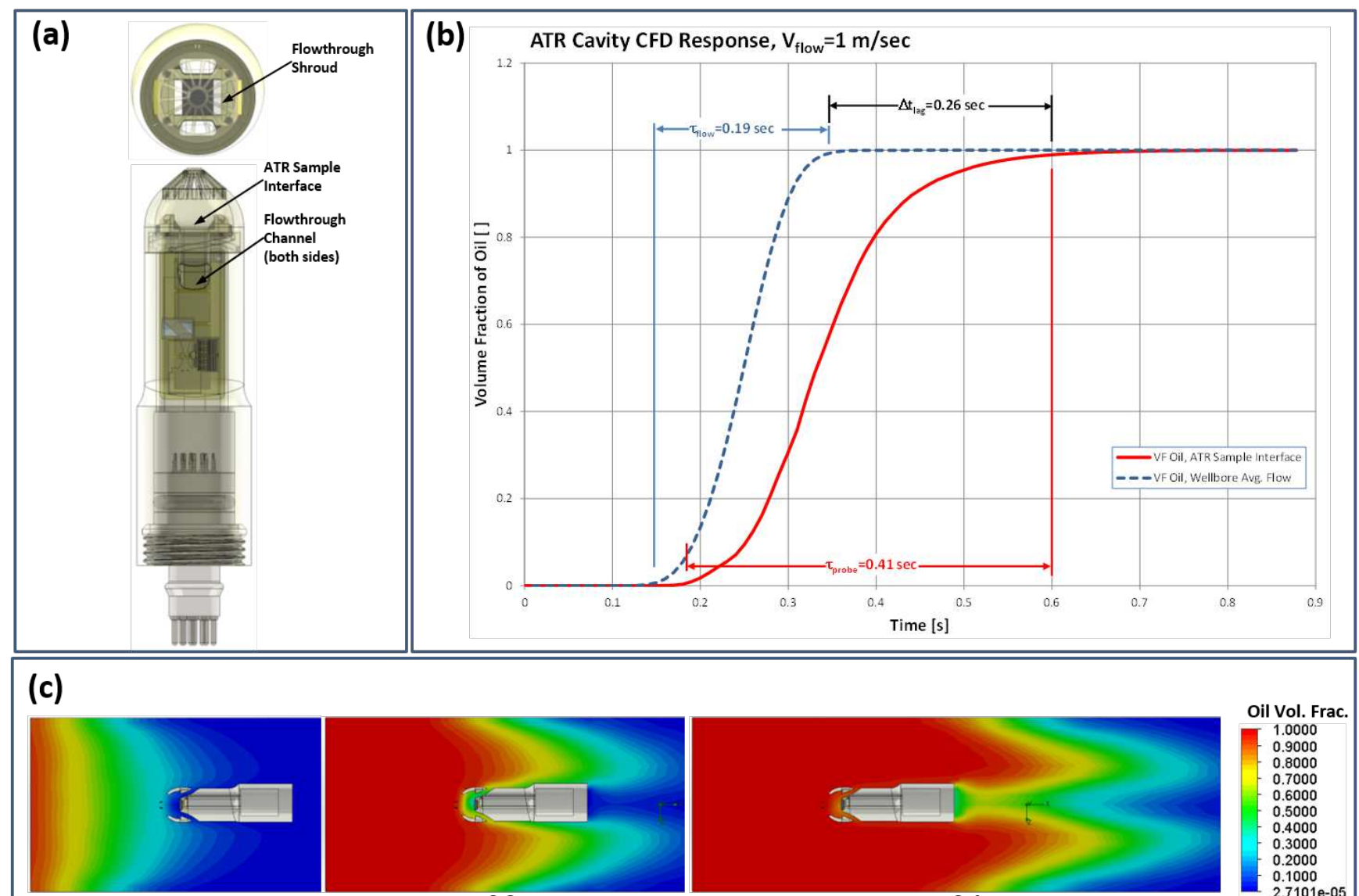

$t=0.2 \mathrm{sec}$
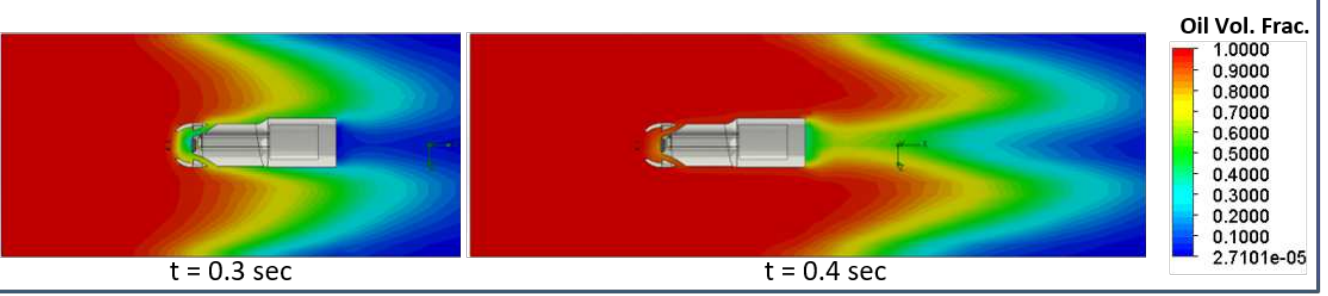

Figure 15. FT-MIR Probe fluid dynamics simulations, $V_{\text {flow }}=1 \mathrm{~m} / \mathrm{sec}$. (a) Flow sampling design, (b) ATR sample interface response to water-to-oil transition, (c) graphical illustration of water-to-oil transition through ATR sampling cavity.

surrounding wellbore fluid was evaluated from CFD simulation response using a notional scenario having a mean wellbore flow rate of $1 \mathrm{~m} / \mathrm{sec}$, initially comprised solely of fresh water then transitioning to a light crude oil very rapidly within $0.1 \mathrm{sec}$. The transient response of the oil volume fraction on the ATR sampling surface and the average wellbore flow is shown in Figure $15 \mathrm{~b}$. The results indicate at the probe cross-section the transition time ( $99 \%$ oil volume fraction) for the wellbore flow $\tau_{\text {flow }}=0.19 \mathrm{sec}$ and the ATR sample interface transition time $\tau_{\text {probe }}=0.41 \mathrm{sec}$, with approximately $0.26 \mathrm{sec}$ differential in the time to reach $99 \%$ oil fraction. For this moderately rapid flow rate of $1 \mathrm{~m} / \mathrm{sec}, 0.26 \mathrm{sec}$ time differential for probe measurement lag is considered very acceptable for downhole real time measurement. A graphical illustration of the water-to-oil transition is shown in the contour plots of Figure 15c. 


\section{Conclusion}

In this work we report a miniaturized FT-MIR spectroscopic probe adaptable to downhole chemical analysis applications. The fundamental development lies within the use of a complex sub-cellular geometry metasurface to develop "FT-MIR on-achip." The metasurface forms the basis of an uncooled microbolometer and a band-limited thermal infrared source that allow circumvention of the limiting technological constraints that have prevented migration of conventional systems to downhole. The metasurface cellular pattern is derived from a geometric inversion of the canonical TanCirc conformal mapping contours and was found to exhibit a near zero index metamaterial (NZIM) behavior. The near-zero-index properties of the metasurface are hypothesized to associate the absorption phenomenom with surface plasmon resonances that confine the absorption mechanism within the ultrathin $(\lambda / 300)$ metasurface plane. The resulting absorption properties of the microbolometer design were found to be relatively insensitive to moderate changes in the material properties of the remaining laminae of the microbolometer. This unusual feature allows the metasurface to be integrated on a single $\mathrm{VO}_{2}$ material thermometric layer which can then be operated at downhole elevated temperatures within the $\mathrm{VO}_{2}$ metal-insulator-transition region. Within this region the $\mathrm{VO}_{2}$ layer exhibits more than an order of magnitude enhancement in its ambient thermometric properties. This leads to an uncooled microbolometer design with predicted maximum detectivity $\mathrm{D}^{*}=1.5 \times 10^{10} \mathrm{~cm} \sqrt{\mathrm{Hz}} / \mathrm{W}$ and noise equivalent difference temperature NEDT of $1 \mathrm{mK}$ at a modulation frequency of $500 \mathrm{~Hz}$, compatible with downhole measurement rates exceeding 2 $\mathrm{Hz}$. A sub-millimeter scale thermal infrared source with intrinsic mid-infrared bandwidth limited emission is formed from the same cellular geometric building block with a predicted irradiance of $630 \mathrm{~mW} / \mathrm{cm}^{2}$ from a $68 \%$ FWHM spectrum centered on $1400 \mathrm{~cm}^{-1}$, using a substrate temperature of $627^{\circ} \mathrm{C}$. The spectrometer integrates a MEMS based Michelson-type interferometer built around the uncooled metasurface microbolometer and a moveable mirrors displacement amplification mechanism creating a 9.4:1 advantage which is calculated to deliver a spectral resolution of $10 \mathrm{~cm}^{-1}$ over the mid-IR range up to $2000 \mathrm{~cm}^{-1}$. This is compatible with the performance found in standard laboratory grade spectroscopic instruments having spectral resolutions between $4-16 \mathrm{~cm}^{-1}$ in the mid-IR bandwidth and could represent a significant step in the effort towards deploying miniaturized uncooled mid-infrared spectroscopy into oilfield downhole logging applications.

\section{Data Availability}

All data analyzed during this study are included in this published article.

\section{Competing Interests}

The author declares no competing interests. 


\section{References}

1. Sabry, Y. M. et al. Ultra-compact mems ftir spectrometer. In Proceedings of SPIE 10210, Next-Generation Spectroscopic Technologies $X$ (2017).

2. Zheng, S., Cai, H., Gu, Y., Chin, L. K. \& Liu, A. Q. High-resolution on-chip spectrometer with a tunable micro-ring resonator filter. Conf. Lasers ElectroOpt. 2 AM1J.2, 1-2 (2016).

3. Souza, M. C. M. M., Grieco, A., Frateschi, N. C. \& Fainman, Y. Fourier transform spectrometer on silicon with thermo-optic non-linearity and dispersion correction. Nat. Commun. 9, 1-8 (2018).

4. Kita, D. M. et al. High-performance and scalable on-chip difital fourier transform spectroscopy. Nat. Commun. 9, 1-7 (2018).

5. Moon, P. H. \& Spencer, D. E. Field Theory Handbook (Springer-Verlag, 1988), 2 edn.

6. Swett, D. W. Near zero index perfect metasurface absorber using inverted conformal mapping. Sci. Reports 10, 9731 (2020).

7. Zeman, E. J. \& Schatz, G. C. An accurate electromagnetic theory study of surface enhancement factors fo ag, au, cu, li, na, al, ga, in, zn, and cd. Am. Chem. Soc. 91, 634-643 (1987).

8. Smith, D. R., Vier, D. C., Koschny, T. \& Soukoulis, C. M. Electromagnetic parameter retrieval from inhomogeneous metamaterials. Phys. Rev. E 71, 036617 (2005).

9. Markel, V. A. Can the imaginary part of permeability be negative? Phys. Rev. E 78, 026608 (2008).

10. Arslanagic, S. et al. A review of the scattering-parameter extraction method with clarification of ambiguity issues in relation to metamaterial homogenization. IEEE Antennas Propag. Mag. 55, No. 2, 91-106 (2013).

11. Jin, Y., Xiao, S., Mortensen, N. A. \& He, S. Arbitrarily thin metamaterial structure for perfect absorption and giant magnification. Opt. Express 19, No. 12, 11114-11119 (2011).

12. Feng, S. \& Halterman, K. Perfect absorption in ultrathin epsilon-near-zero metamaterials induced by fast-wave non-radiative modes. Phys. Rev. B 86, 165103 (2012).

13. Zhong, S., Ma, Y. \& He, S. Perfect absorption in ultrathin anisotropic $\varepsilon$-near-zero metamaterials. Appl. Phys. Lett. 105, 023504 (2014).

14. Raether, H. Surface Plasmons on Smooth and Rough Surfaces and on Gratings (Springer-Verlag, 1986), 1 edn.

15. Takami, H., Kawatani, K., Kanki, T. \& Tanaka, H. High temperature-coefficient of resistance at room temperature in w-doped vo2 thin films on al2o3 substrate and their thickness dependence. Jpn. J. Appl. Phys. 50, 055804 (2011).

16. Hamamatsu Corp. Characteristics and use of infrared detectors. Technical Information SD-12, Cat. No. KIRD9001E04.

17. Pergament, A. L., Crunteanu, A. \& Beaumont, A. Vanadium dioxide: Metal-insulator transition, electrical switching and oscillations, a review of state of the art and recent progress. In Energy Materials and Nanotechnology (EMN) Meeting on Computation and Theory (2015).

18. Aske, N., Kallevik, H. \& Sjoblom, J. Determination of saturate, aromatic, resin, and asphaltenic (sara) components in crude oils by means of infrared and near-infrared spectroscopy. Energy \& Fuels 15, 1304-1312 (2001).

19. Muhlstein, C. L., Brown, S. B. \& Ritchie, R. O. High-cycle fatigue of single-crystal silicon thin films. J. Microelectromechanical Syst. 10-4, 593-600 (2001). 